\title{
TRATAMIENTO DE MEMBRANAS NEOVASCULARES MIÓPICAS CON RANIBIZUMAB
}

\section{RANIBIZUMAB AS TREATMENT FOR MYOPIC CHOROIDAL NEOVASCULARIZATION}

\author{
TORRÓN C ${ }^{1}$, EGEA C ${ }^{2}$, FERRER E ${ }^{1}$ RUIZ O $^{1}$, HONRUBIA FM ${ }^{1}$
}

\begin{abstract}
RESUMEN
Objetivo: Determinar la eficacia de Ranibizumab intravítreo, como tratamiento de la neovascularización coroidea asociada a miopía patológica.

Material y métodos: Estudio retrospectivo, no comparativo de 18 ojos tratados con inyecciones intravítreas de Ranibizumab. Diez de los casos se habían tratado previamente con Terapia Fotodinámica, los 8 restantes recibieron Ranibizumab como primera terapia. Tras realizar angiografía fluoesceínica (AGF) y tomografía de coherencia óptica (OCT), se inyectaba ranibizumab, en los controles mensuales posteriores y teniendo en cuenta la agudeza visual (AV), presencia de metamorfopsias, biomicroscopia de polo posterior y características de la OCT se decidía el retratamiento.

Resultados: Se analizaron 18 ojos de 16 pacientes, con un seguimiento mínimo de 6 meses. La edad media fue de 56,4 años y el equivalente esférico medio de $-13,3$ dioptrias. Todas las membranas neovasculares fueron angiográficamente clásicas y de localización sub o yuxtafoveal. La AV mejoró en 14 ojos $(77,7 \%)$ al menos 1 línea y 11 ojos $(61,1 \%)$ mejoraron 2 o más líneas a los 6 meses de la inyección, 3 ojos no mostraron cambio $(16,6 \%)$ y sólo 1 empeoró 1 línea $(5,5 \%)$. A los 6 meses la AV media mejoró de 0,25 a 0,46 ( $\mathrm{p}=$ 0,001). La media del espesor macular en la OCT se redujo de 344,9 micras a $212,6(p=0,015)$.
\end{abstract}

\begin{abstract}
Objective: To evaluate the efficacy of intravitreal Ranibizumab as treatment for choroidal neovascularisation due to pathological myopia.

Materials and methods: A retrospective, noncomparative study of 18 eyes treated with intravitreal injections of Ranibizumab.

Ten eyes had been treated previously with photodynamic therapy and eight received Ranibizumab as first therapy. After thorough ophthalmologic examination, fluorescein angiography (FAG) and optical coherence tomography (OCT), intraocular injection of Ranibizumab was performed. In subsequent monthly follow ups and taking into account visual acuity, presence or absence of metamorphopsia, biomicroscopy and OCT examination, further treatment was decided.

Results: Eighteen eyes from 16 patients were finally included. Patients were followed up for a minimum of 6 months. The mean age at initial treatment was 56.4 years. Mean refractive error was -13.3 diopters. Regarding FAG, all neovascular membranes were classical and sub or juxtafoveal localised. At the end of the sixth month after treatment fourteen eyes $(77.7 \%)$ showed better visual acuity ranging from one or more lines on the Snellen chart, eleven eyes $(61.1 \%)$ improved two lines or more, three eyes $(16.6 \%)$ did not show any change and one eye $(5.5 \%)$ worsened by one line. At 6 months the mean best-corrected visual acuity improved from 0.25 to
\end{abstract}

\footnotetext{
Recibido: 21/7/08. Aceptado: 13/10/09.

Servicio de Oftalmología. Hospital Universitario Miguel Servet. Zaragoza. España.

${ }^{1}$ Doctor en Medicina.

2 Licenciada en Medicina.

Correspondencia:

Clemencia Torrón

Vía Hispanidad, $35,1 .^{\circ} \mathrm{A}$

50012 Zaragoza

España

E-mail: ctorron@salud.aragon.es
} 
Conclusiones: Ranibizumab puede ser una buena opción terapéutica en el tratamiento de la neovascularización miópica, con mejoría visual y anatómica, incluso en pacientes no respondedores a Terapia Fotodinámica.

Palabras clave: Miopía patológica, ranibizumab, Lucentis, antiangiogénicos, neovascularización. $0.46(\mathrm{p}=0.001)$. The mean central macular thickness decreased from 344.9 to 212.6 ( $\mathrm{p}=0.015)$.

Conclusions: Ranibizumab may be a good therapeutic option as treatment for choroidal neovascularisation due to pathological myopia; it improved visual acuity and anatomical features, even in nonresponders to photodynamic therapy (Arch Soc Esp Oftalmol 2009; 84: 507-514).

Key words: Pathological myopia, ranibizumab, Lucentis, antiangiogenic, neovascularisation.

\section{INTRODUCCIÓN}

La maculopatía miópica, es una de las principales causas de ceguera irreversible en el mundo, afectando también a pacientes jóvenes y de mediana edad. En la mayoría de los casos, la pérdida de visión está ocasionada por la atrofia coriorretiniana o por el desarrollo de una neovascularización coroidea (NVC) (1-3). Generalmente, las neovascularizaciones miópicas son más frecuentes en mujeres, se relacionan con la presencia de grietas lacadas, son angiográficamente clásicas y de tamaño menor a un diámetro de disco (1-5).

Actualmente, el único tratamiento aprobado para la NVC asociada a miopía patológica es la Terapia Fotodinámica (TFD) con verteporfina. La TFD ha demostrado estabilización de la visión en los ojos tratados, en comparación con el grupo placebo (46); sin embargo, la aparición de un nuevo grupo de fármacos, llamados antiangiogénicos, y su utilización creciente en el tratamiento de la Degeneración Macular Asociada a la Edad (DMAE), al demostrar no sólo estabilización sino mejorías de agudeza visual (7-11), ha abierto una nueva vía en el manejo terapéutico de los pacientes con NVC miópica. En los últimos 3 años, se han publicado algunos casos clínicos y pequeñas series de casos de pacientes con membranas miópicas tratados con Bevacizumab $\left(\right.$ Avastin $\left.^{\circledR}\right)$, tanto por vía sistémica como intraocular, con resultados prometedores (12-25). Bevacizumab, es un anticuerpo recombinante humanizado que bloquea todas las isoformas del Factor de Crecimiento del Endotelio Vascular («VEGF»). Sin embargo, este fármaco no está aprobado para uso intraocular como Ranibizumab (Lucentis ${ }^{\circledR}$ ). Ranibizumab es una fracción Fab de anticuerpo recombinante humanizado que, como Bevacizumab, bloquea todas las formas activas del
«VEGF». En estudios randomizados, doble ciego ha demostrado su eficacia en uso intravítreo para el tratamiento de cualquier tipo de membrana neovascular, mejorando sustancialmente los resultados de la TFD y consiguiendo mejorías de agudeza visual en un porcentaje importante de pacientes con DMAE (7-9).

En esta pequeña serie retrospectiva de casos, presentamos los resultados del tratamiento con Ranibizumab en pacientes con membranas miópicas.

\section{SUJETOS, MATERIAL Y METODOS}

Presentamos un estudio, para evaluar la respuesta de 18 ojos diagnosticados de NVC miópica en los que se ha realizado tratamiento con Ranibizumab intravítreo en nuestro Servicio.

En todos los casos se realizó en cada visita una exploración oftalmológica completa que incluía: AV (optotipos standard tipo Snellen), biomicroscopía de polo anterior, tonometría por aplanación, exploración de fondo de ojo con oftalmoscopio binocular, biomicroscopía de polo posterior con lente de 78 dioptrías y examen cualitativo y cuantitativo macular mediante el Stratus OCT 3000 (Zeiss Meditec, Jena-Germany). La angiografía con fluoresceína (AGF) se realizó a todos los pacientes antes de iniciar el tratamiento y posteriormente, en los casos que se consideró necesario.

Los pacientes fueron informados verbalmente y por escrito de las características del tratamiento y su utilización en uso compasivo, firmando el consentimiento. Se prescribió un colirio antibiótico (Ciprofloxacino, Oftacilox ${ }^{\circledR}$, Alcon-Cusí, Barcelona, España), que debían utilizar 4 veces al día los 3 días previos a la inyección intraocular y los 4 días posteriores a la misma. 
Previamente a la inyección se instiló en 2 ocasiones colirio de anestesia (oxibuprocaína al 0,4\% y tetracaína al 0,5: Colircusí anestésico doble ${ }^{\circledR}$, Alcon-Cusí, Barcelona, España), y povidona yodada al $5 \%$. Se inyectaron $0,05 \mathrm{ml}$ de Ranibizumab $0,5 \mathrm{mg}$ (Novartis, West Sussex,Reino Unido), en el cuadrante inferotemporal, a 3,5-4 $\mathrm{mm}$ del limbo. Los pacientes fueron advertidos de acudir a urgencias en caso de notar anomalías en su visión, dolor u otra complicación.

Las revisiones se realizaban a las 4 semanas, repitiendo la inyección siempre que fuera necesario, por persistencia en OCT de líquido intra o subretiniano, desprendimiento de epitelio pigmentario (DEP), aparición de hemorragia, nueva NVC o crecimiento de la ya existente. En caso de duda, además de los signos clínicos, consideramos la persistencia de metamorfopsias a la hora de decidir el retratamiento.

Se comprobó el ajuste a la normalidad de todas las variables analizadas mediante un test de Kolmogorov-Smirnov. Se compararon los resultados entre grupos mediante el test t-Student. El programa informático utilizado fue el SPSS versión 15.

\section{RESULTADOS}

Se analizaron 18 ojos de 16 pacientes, siete hombres y nueve mujeres. La edad media ha sido 56,44 años (rango 33-78), el equivalente esférico medio ha sido de $-13,36$ dioptrías, (rango -6 a -24). El seguimiento medio ha sido de 7,5 meses (rango 6$12)$, con una media de inyecciones de Lucentis ${ }^{\circledR}$ de 2,61 (rango 1-6).

En la tabla I se describen las características basales de los pacientes.

En 10 ojos se habían realizado tratamientos previos: 8 ojos recibieron anteriormente TFD, un ojo se trató con láser por membrana extrafoveal antes de la TFD (recidiva subfoveal).En estos pacientes, la inyección de Lucentis ${ }^{\circledR}$ se realizó a partir del tercer mes de realizada la TFD.Un ojo se trató con TFD y 1 inyección de Avastin $^{\circledR}$, en este caso se inyectó Lucentis ${ }^{\circledR}$ a los 2 meses de la inyeción de Avastin $^{\circledR}$.

En la tabla II se exponen la media y desviación estándar de AV y espesor macular central en la OCT al mes, 3 meses, 6 meses y final del seguimiento.

La AV inicial media (DS) fue de $0,25(0,17)$, a los 6 meses fue de $0,46(0,3)$, diferencia muy significativa $(\mathrm{p}=0,001)$. La AV media final fue de 0,44 $(0,26)$ con una ganancia media de dos líneas. En 14 ojos $(77,7 \%)$ se observó mejoría de al menos una línea de agudeza visual y 11 ojos $(61,1 \%)$ mejoraron dos o más líneas, tres ojos permanecieron igual $(16,6 \%)$ y sólo un ojo empeoró una línea $(5,5 \%)$ a los 6 meses de la inyección de Lucentis ${ }^{\circledR}$.

El espesor macular central (DS) medio inicial fue de 344,93 micras $(202,90)$, con reducción significa-

Tabla I. Características basales del grupo estudiado, tiempo de seguimiento, tipo y localización de la membrana, tratamientos previos y número de inyecciones de Lucentis

\begin{tabular}{|c|c|c|c|c|c|c|c|}
\hline Ojos & Sexo & Edad & Equivalente esférico & Tiempo seguimiento & Tipo membrana & Tratamiento previo & N. ${ }^{o}$ lucentis \\
\hline 1 & $\mathrm{~V}$ & 73 & -11 & 7 & Clásica/subf. & Sí laser TFD & 4 \\
\hline 2 & M & 40 & -15 & 6 & Clásica /subf. & No & 2 \\
\hline 3 & M & 36 & -24 & 9 & Clásica/yuxt. & No & 2 \\
\hline 4 & M & 59 & -13 & 6 & Clásica/subf. & No & 3 \\
\hline 5 & $\mathrm{~V}$ & 51 & -17 & 6 & Clásica/subf. & No & 1 \\
\hline 6 & $\mathrm{~m}$ & 46 & -13 & 6 & clásica/subf. & No & 2 \\
\hline 7 & M & 76 & -12 & 8 & Clásica/subf. & No & 6 \\
\hline 8 & $\mathrm{~V}$ & 56 & -6 & 7 & Clásica/subf. & No & 4 \\
\hline 9 & $\mathrm{~V}$ & 43 & $-13,50$ & 6 & Clásica/yuxt. & No & 1 \\
\hline 10 & M & 77 & -13 & 9 & Clásica/subf. & Sí TFD & 3 \\
\hline 11 & $\mathrm{~V}$ & 61 & -9 & 6 & Clásica/subf. & Sí TFD & 1 \\
\hline 12 & V & 61 & -10 & 7 & Clásica/subf. & Sí TFD & 1 \\
\hline 13 & M & 39 & -17 & 6 & Clásica/subf. & Sí TFD & 2 \\
\hline 14 & M & 33 & -13 & 12 & Clásica/subf. & Sí TFD avastin & 4 \\
\hline 15 & $\mathrm{~V}$ & 56 & -20 & 12 & Clásica/yuxt. & Sí TFD & 3 \\
\hline 16 & $\mathrm{~V}$ & 56 & $-18,75$ & 6 & Oculta/yuxt. & Sí TFD & 2 \\
\hline 17 & $\mathrm{~V}$ & 78 & $-6,75$ & 6 & Clásica/subf. & Sí TFD & 4 \\
\hline 18 & M & 75 & $-8,5$ & 10 & Clásica/subf. & Sí TFD & 2 \\
\hline
\end{tabular}

V: varón; M: mujer; subf: subfoveal; yuxt: yuxtafoveal. 
Tabla II. Agudeza visual (AV) y espesor macular central: media y desviación estándar (DS) basal, 1, 3, 6, meses y al final del seguimiento

\begin{tabular}{lccccc}
\hline & Basal & 1 mes & 3 meses & 6 meses & Final \\
\hline Parámetros visuales AV (Snellen) & $0,251(0,17)$ & $\begin{array}{c}0,413(0,26) \\
0,006^{*}\end{array}$ & $\begin{array}{c}0,392(0,25) \\
0,026^{*}\end{array}$ & $\begin{array}{c}0,464(0,30) \\
0,001^{*}\end{array}$ & $0,442(0,26)$ \\
\hline $\begin{array}{l}\text { OCT: Espesor macular central } \\
\text { en micras }\end{array}$ & $344,93(202,90)$ & $\begin{array}{c}246,37(86,80) \\
0,015^{*}\end{array}$ & $\begin{array}{c}221,15(100,19) \\
0,008^{*}\end{array}$ & $\begin{array}{c}212,6(74,02) \\
0,015^{*}\end{array}$ & $219,33(58,32)$ \\
\hline \hline
\end{tabular}

* Diferencia estadísticamente significativa con $\mathrm{p}<0,05$.

Tabla III. Comparación de las medias a los 6 meses de agudeza visual (AV), espesor macular central en tomografía de coherencia óptica (OCT) y número de inyecciones entre pacientes sin y con Terapia Fotodinámica (TFD) previa

\begin{tabular}{lccc}
\hline Parámetro & SIN TFD & CON TFD & $\mathrm{p}$ \\
\hline $\mathrm{AV}$ & 0,538 & 0,406 & 0,383 \\
OCT & 216,7 & 209 & 0,849 \\
Número inyecciones & 2,18 & 2,60 & 0,507 \\
\hline \hline
\end{tabular}

* Diferencias estadísticamente significativas con $\mathrm{p}<0,05$.
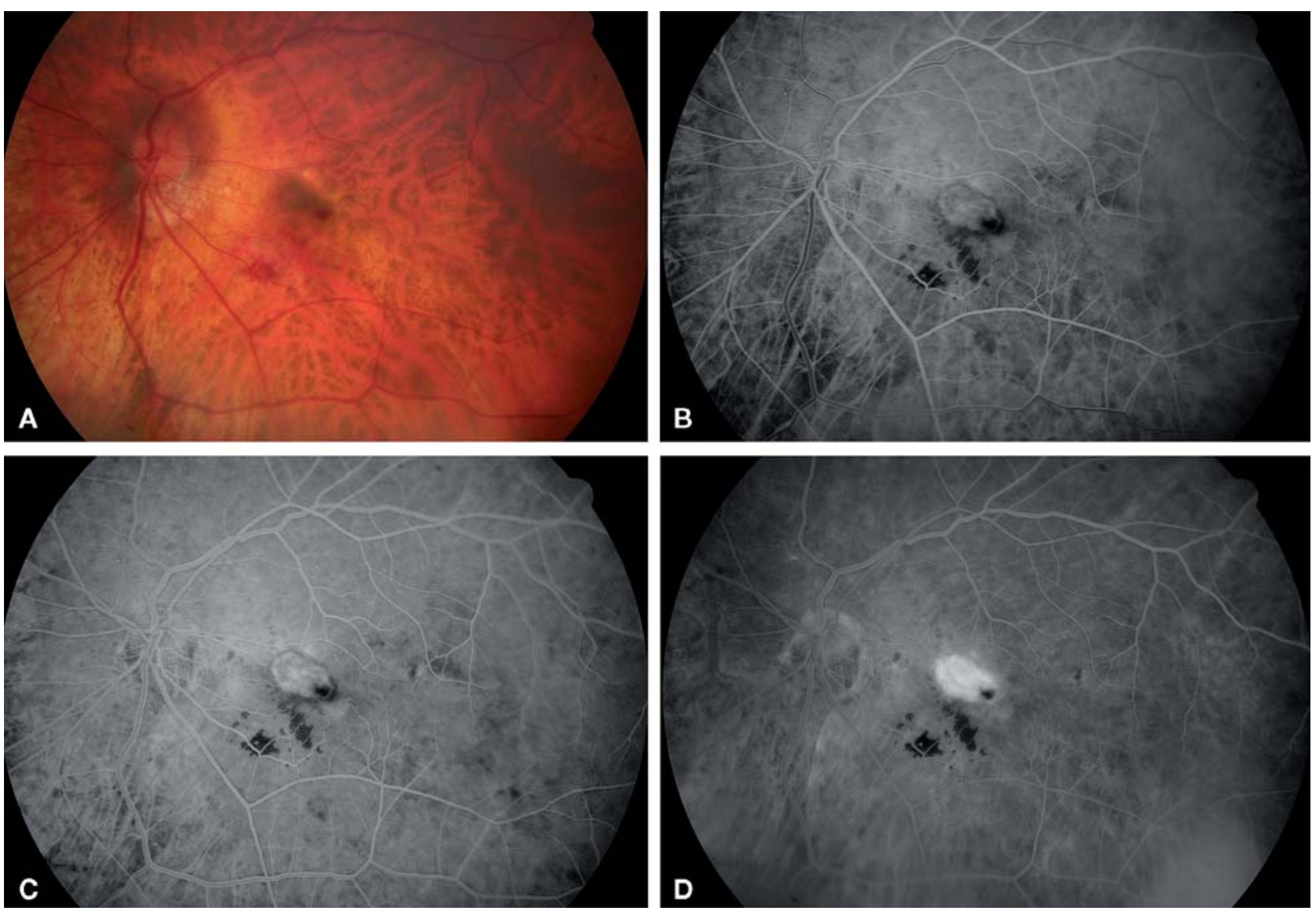

Fig. 1: Caso 4: Mujer de 59 años, 3 inyecciones de ranibizumab, con un seguimiento de 6 meses. AV inicial 0,12, AV 6 meses 0,3. A: Retinografía; B, C y D: Angiografía: Membrana clásica subfoveal activa. 

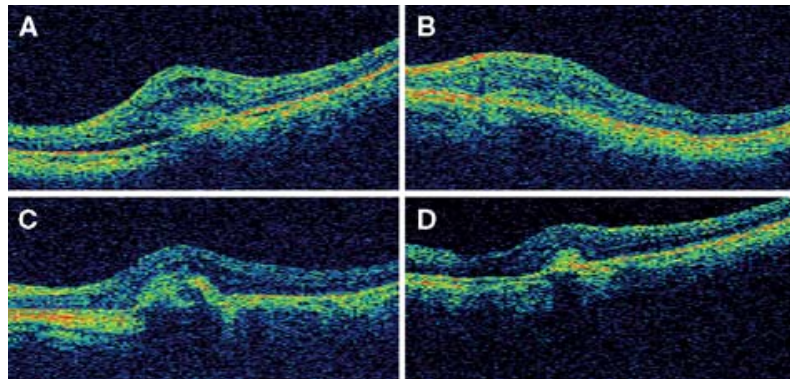

Fig. 2: Tomografía de coherencia óptica A: inicial; B: al mes; C: a los 3 meses; D: a los 6 meses. Espesor central inicial $775 \mu \mathrm{m}$ a los 6 meses $356 \mu \mathrm{m}$.

Del mismo modo, comparando los pacientes menores de 50 años de edad con los de más de 50 años, observamos que no había diferencias estadísticamente significativas en cuanto a la AV y al número de inyecciones, sin embargo el espesor macular central en la OCT era significa-
Tabla IV. Comparación de medias a los 6 meses de AV, espesor macular central en tomografía de coherencia óptica OCT y número de inyecciones entre pacientes menores y mayores de $\mathbf{5 0}$ años

\begin{tabular}{lccc}
\hline Parámetro & $\begin{array}{c}\text { Pacientes } \\
<50 \text { años }\end{array}$ & $\begin{array}{c}\text { Pacientes } \\
>50 \text { años }\end{array}$ & $\mathrm{p}$ \\
\hline AV & 0,617 & 0,388 & 0,141 \\
OCT & 169,33 & 241,44 & $0,034^{*}$ \\
Número inyecciones & 2 & 2,57 & 0,39 \\
\hline \hline
\end{tabular}

* Diferencias estadísticamente significativas con $\mathrm{p}<0,05$.

tivamente mayor en el grupo de más de 50 años (tabla IV).

No hemos observado complicaciones sistémicas o locales derivadas del tratamiento. En un caso hubo una recidiva de la NVC, con sangrado y disminución de AV con respecto al mejor valor obtenido, pero manteniendo la AV inicial (caso 7).
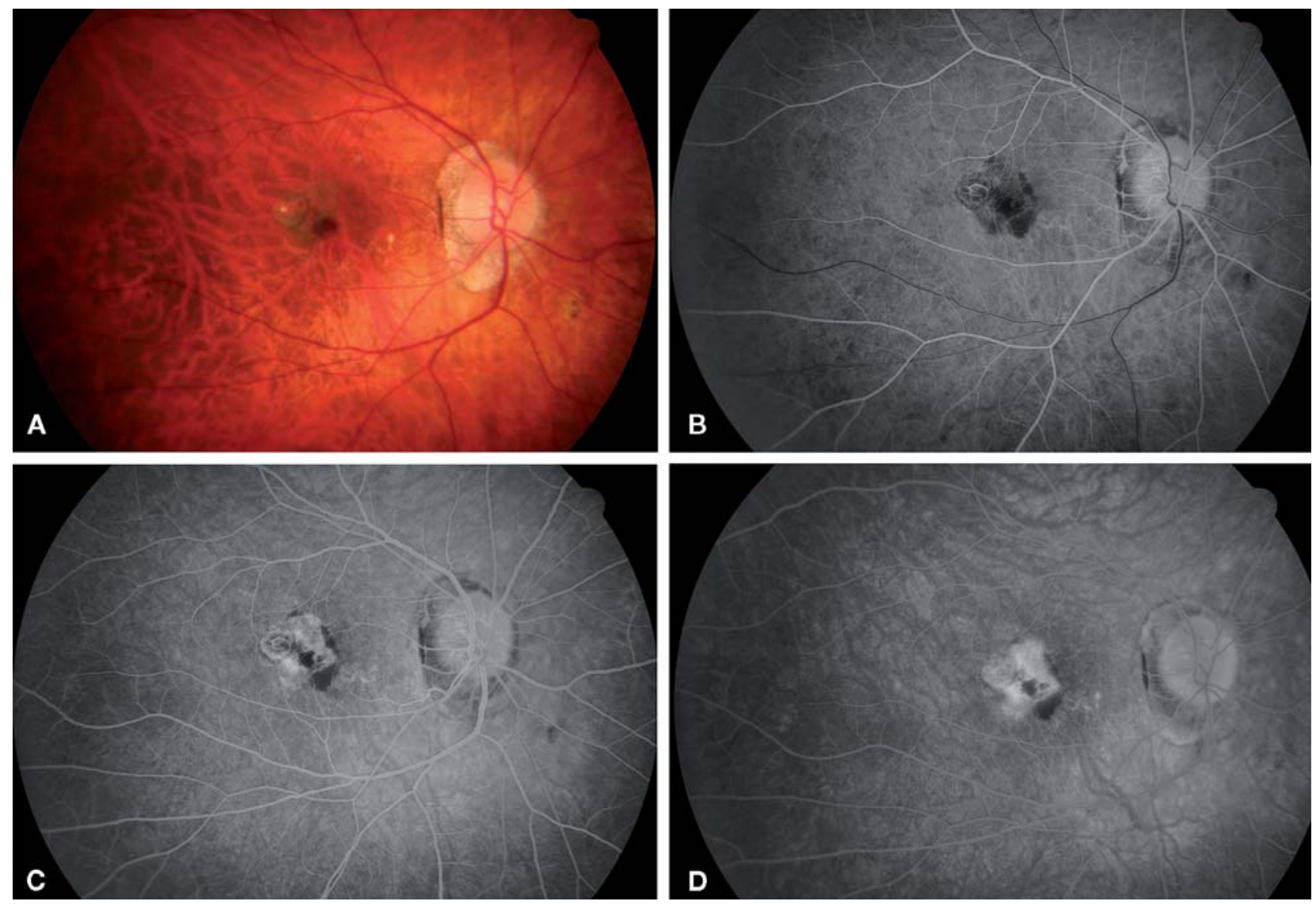

Fig. 3: Caso 14: Mujer de 33 años, tratamiento previo con TFD y una inyección de Avastin, 4 inyecciones de ranibizumab, AV inicial 0,3, a los 6 meses 1. A: Retinografía; B, C y D: Angiografía: Membrana clásica subfoveal activa, en borde de cicatriz. 

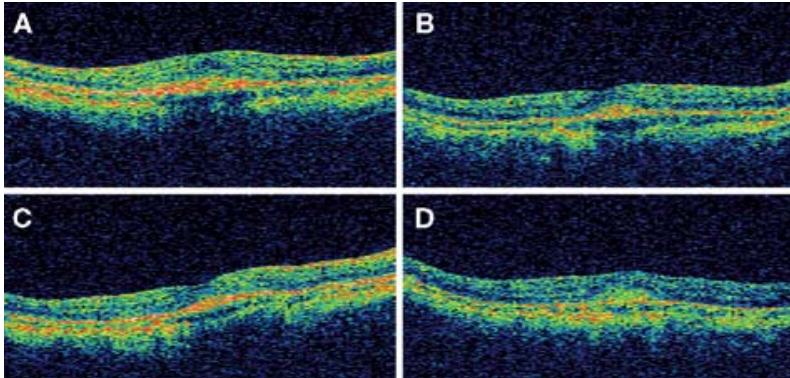

Fig. 4: Tomografía de coherencia óptica A: inicial; B: al mes; C: a los 3 meses; D: a los 6 meses. Espesor inicial $258 \mu \mathrm{m}$, a los 6 meses $167 \mu \mathrm{m}$.

\section{DISCUSION}

El tratamiento de elección de la NVC secundaria a miopía patológica, ha sido durante los últimos años la TFD con verteporfina. Esta terapia, con resultados prometedores inicialmente, no ha conseguido en estudios a largo plazo evitar la pérdida de visión en los pacientes afectados $(4,5)$. En el estudio VIP a los 2 años de seguimiento, $36 \%$ de los ojos tratados vs $51 \%$ del grupo placebo perdieron al menos 8 letras de agudeza visual, cambio no significativo. En el mismo estudio $40 \%$ de los ojos tratados vs $13 \%$ del grupo placebo mejoraron al menos 5 letras y únicamente, $12 \%$ de los ojos tratados vs 0\% de los del grupo placebo mejoraron 3 líneas de visión, resultando esta diferencia significativa (5).

Estos resultados y la experiencia obtenida en DMAE con el nuevo grupo de fármacos antiangiogénicos ha llevado a algunos autores a utilizarlos en la terapia de la NVC miópica. El fármaco más utilizado es el Avastin ${ }^{\circledR}$, en tratamiento tanto de rescate, por fracaso de la TFD, como de primera elección. Nguyen (12), publicó en 2005 el primer artículo sobre el uso de Avastin ${ }^{\circledR}$ intravenoso en 2 pacientes con NVC miópica en los que habían fracasado varias sesiones de TFD, con espectacular resultado. Laud (18), publicó poco después el primer artículo sobre Avastin ${ }^{\circledR}$ intravítreo en esta patología. Otros autores han publicado posteriormente pequeñas series y casos clínicos sobre Avastin ${ }^{\circledR}$ en membranas miópicas (13-25). Todos los autores parecen coincidir en los prometedores resultados del tratamiento aunque son series cortas, no existe grupo control y el seguimiento es escaso. Hasta el momento, existen sólo 4 publicaciones sobre los resultados de Lucentis ${ }^{\circledR}$ en NVC miópica (26-29). La razón reside, probablemente, en que al ser ambos de «uso compasivo» se ha optado por el fármaco más económico, aproximadamente 20 euros la dosis de Avastin $^{\circledR}$ frente a los más de 1.200 euros de la inyección de Lucentis $^{\circledR}$. Como reconoce Rosenfeld (30), existe en este momento una importante controversia acerca de si es ético el uso «off label» de Avastin ${ }^{\circledR}$ como tratamiento primario de la NVC miópica, cuando la TFD está aprobada para el tratamiento de éstos pacientes.

En nuestro hospital, la decisión de iniciar el tratamiento con Lucentis en NVC miópica, vino en relación con el fracaso de la TFD para conseguir inactivar la lesión en algunos pacientes. Los resultados obtenidos en estos casos nos animaron a tratar otros pacientes de primera intención, con el objetivo de mejorar los resultados visuales de la TFD.

En nuestra serie de 18 ojos, 14 ojos $(77,7 \%)$ mejoraron al menos una línea de AV y 11 ojos $(61,1 \%)$ mejoraron dos o más líneas a los 6 meses de la inyección de Lucentis ${ }^{\circledR}$, tres ojos permanecieron igual $(16,6 \%)$ y sólo un ojo empeoró una línea $(5,5 \%)$. La AV media inicial fue de 0,23 , mientras que la AV media final fue de 0,44 , con una ganancia media de dos líneas de AV, resultados comparables a los de Monés (29) pero inferiores a los de Silva (26), Konstantinidis (28) y Lai (27), con mejoría media entre 2,5 y 3,8 líneas.

Nuestros resultados, también son similares a los obtenidos por otros autores con Avastin ${ }^{\circledR}$. En la serie de 8 ojos de Sakaguchi (20), 75\% de los ojos, tuvieron una mejoría de 2 líneas o más de AV, permaneciendo el $25 \%$ restante igual; en la serie de 22 ojos de Chan (13), 68,2\% de los ojos mejoraron 2 o más líneas, con un incremento visual medio de 2,6 líneas a los 6 meses y de 2,4 líneas al año (23). Mejor resultado obtienen Yamamoto (15), Gharbiya (24) y Mandal (16) con incrementos entre 3,5 y 5,1 líneas de AV.

Como otros autores (13,15-17,19-29), también hemos encontrado una reducción estadísticamente significativa en el espesor macular central en todos los controles de OCT.

Cuando comparamos los pacientes tratados previamente con los no tratados, la AV media fue mejor y el número de inyecciones menor en el grupo de pacientes no tratados previamente, pero estas diferencias no fueron significativas. Monés (29), en cambio, si encontró mejor resultado visual en los pacientes no tratados con ganancia de 12,7 letras, frente a las 7,4 letras de los tratados previamente. Esta diferencia también se apreció en el estudio de Arias con Avastin (21). 
En el momento actual, el tratamiento de la NVC miópica presenta una serie de incógnitas que deben ser resueltas. En primer lugar si los antiangiogénicos son más eficaces que la TFD, en segundo lugar cual es el antiangiogénico de elección y en tercer lugar cual es el régimen de administración más adecuado.

Los resultados de estas pequeñas series parecen en principio favorables al uso de antiangiogénicos, pero hay que tener muy en cuenta que se trata de series cortas, sin grupo control y escaso seguimiento.

En cuanto al antiangiogénico de elección, mientras no existan estudios comparativos entre ambos fármacos, el diseño para uso intraocular iría a favor de Lucentis ${ }^{\circledR}$, mientras que el menor coste económico iría a favor de Avastin ${ }^{\circledR}$.

Como en el caso de la DMAE, el régimen de administración debe ser independizado en cada paciente para conseguir los mejores resultados con el menor número posible de inyecciones (31-33). En la mayoría de los estudios con Avastin ${ }^{\circledR}$ (15$17,20-22,24)$ y Lucentis $^{\circledR}(26,28-29)$ el régimen terapéutico ha sido, según necesidad del paciente. En nuestro estudio con Lucentis ${ }^{\circledR}$, la media fue de 2,5 inyecciones, similar al de Silva (26) y Konstantinidis (28). En la serie de Monés (29) con un seguimiento mayor, 1 año, el número medio de inyecciones fue 1,5. En la serie más amplia de Ikuno (22), 63 ojos tratados con Avastin $^{\circledR}$ con seguimiento mínimo de un año, la media fue de 2,4 inyecciones, superior a la media de linyección de Arias (21) y Yamamoto (15) aunque con un seguimiento de 6 meses. Chan $(13,23)$ y Ruiz-Moreno $(25)$, en cambio, proponen un régimen de 3 inyecciones de Avastin ${ }^{\circledR}$ para consolidar el efecto y tratamiento adicional en los casos que sea necesario, al igual que Lai (27) con Lucentis ${ }^{\circledR}$.

En conclusión, creemos, que a pesar de los prometedores resultados obtenidos con la inyección intraocular de Lucentis ${ }^{\circledR}$ para el tratamiento de las NVC miópicas, son necesarios estudios más amplios, randomizados y con mayor seguimiento que aclaren éstas y otras incógnitas.

\section{BIBLIOGRAFÍA}

1. Hotchkiss ML, Fine SL. Pathologic myopia and choroidal neovascularization. Am J Ophthalmol 1981; 91: 177-183.

2. Avila MP, Weiter JJ, Jalkh AE, Trempe CL, Pruett RC, Schepens CL. Natural history of choroidal neovascularization in degenerative myopia. Ophthalmology 1984; 91: 1573-1581.
3. Yoshida T, Ohno-Matsui K, Ohtake Y, Takashima T, Futagami S, Baba T, et al. Long-term visual prognosis of choroidal neovascularization in high myopia: a comparison between age groups. Ophthalmology 2002; 109: 712-719.

4. Verteporfin in Photodynamic Therapy Study Group. Photodynamic therapy of subfoveal choroidal neovascularization in pathologic myopia with verteporfin. 1 year results of a randomized clinical trial-VIP report $n^{\circ}{ }^{\circ} 1$. Ophthalmology 2001; 108: 841-852.

5. Verteporfin in Photodynamic Therapy Study Group. Photodynamic therapy of subfoveal choroidal neovascularization in pathologic myopia with verteporfin. 2 year results of a randomized clinical trial-VIP report $n^{\circ}{ }^{\circ} 3$. Ophthalmology 2003; 110: 667-673.

6. Sickenberg M, Schmidt-Erfurth U, Miller JW, Pournaras $C J$, Zografos L, Piguet E, et al. A preliminary study of photodynamic therapy using verteporfino for choroidal neovascularization in pathologic myopia, ocular histoplasmosis syndrome, angioid streaks and idiopathic causes. Arch Ophthalmol 2000; 117: 327-336.

7. Brown DM, Kaiser PK, Michels M, Soubrane G, Heier JS, Kim RY, et al. Ranibizumab versus verteporfin for neovascular age-related macular degeneration. $N$ Engl J Med 2006; 355: 1432-1444.

8. Rosenfeld PJ, Brown DM, Heier JS, Boyer DS, Kaiser PK, Chung CY, et al. Ranibizumab for neovascular age-related macular degeneration. N Engl J Med 2006; 355: 14191431 .

9. Heier JS, Antoszyk AN, Pavan PR, Leff SR, Rosenfeld PJ, Ciulla TA, et al. Ranibizumab for treatment of neovascular age-related macular degeneration: a phase I/II multicenter, controlled, multidose study. Ophthalmology 2006; 113: 633-642.

10. Avery RL, Pieramici DJ, Rabena MD, Castellarin AA, Nasir MA, Giust MJ. Intravitreal bevacizumab (Avastin) for neovascular age-related macular degeneration. Ophthalmology 2006; 113: 363-372.

11. Bashshur ZF, Haddad ZA, Schakal A, Jaafar RF, Saab M, Noureddin BN. Intravitreal bevacizumab for treatment of neovascular age-related macular degeneration: a one-year prospective study. Am J Ophthalmol 2008; 145: 249-256.

12. Nguyen $Q D$, Shah $S$, Tatlipinar S, Do DV, Anden EV, Campochiaro PA. Bevacizumab suppresses choroidal neovascularisation caused by pathological myopia. $\mathrm{Br} J \mathrm{Oph}$ thalmol 2005; 89: 1368-1370.

13. Chan WM, Lai TY, Liu DT, Lam DS. Intravitreal bevacizumab (Avastin) for myopic choroidal neovascularization: six-month results of a prospective pilot study. Ophthalmology 2007; 114: 2190-2196.

14. Nguyen QD, Shah SM, Hafiz G, Do DV, Haller JA, Pili R, et al. Intravenous bevacizumab causes regression of choroidal neovascularization secondary to diseases other than age-related macular degeneration. Am J Ophthalmol 2008; 145: 257-266.

15. Yamamoto I, Rogers AH, Reichel E, Yates PA, Duker JS. Intravitreal bevacizumab (Avastin) as treatment for subfoveal choroidal neovascularisation secondary to pathological myopia. Br J Ophthalmol 2007; 91: 157-160.

16. Mandal S, Venkatesh P, Sampangi R, Garg S. Intravitreal bevacizumab (Avastin) as primary treatment for myopic choroidal neovascularization. Eur J Ophthalmol 2007; 17: 620-626. 
17. Hernández-Rojas ML, Quiroz-Mercado H, Dalma-Weiszhausz, J, Fromow-Guerra J, Amaya-Espinosa A, SolísVivanco A, et al. Short-term effects of intravitreal bevacizumab for subfoveal choroidal neovascularization in pathologic myopia. Retina 2007; 27: 707-712.

18. Laud K, Spaide RF, Freund KB, Slakter J, Klancnik JM Jr. Treatment of choroidal neovascularization in pathologic myopia with intravitreal bevacizumab.Retina 2006; 26: 960-963.

19. Tewari A, Dhalla MS, Apte RS. Intravitreal bevacizumab for treatment of choroidal neovascularization in pathologic myopia. Retina 2006; 26: 1093-1094.

20. Sakaguchi H, Ikuno Y, Gomi F, Kamei M, Sawa M, Tsujikawa $M$, et al. Intravitreal injection of bevacizumab for choroidal neovascularisation associated with pathological myopia. Br J Ophthalmol 2007; 91: 161-165.

21. Arias L, Planas N, Prades S, Caminal JM, Rubio M, Pujol $O$, et al. Intravitreal bevacizumab (Avastin) for choroidal neovascularisation secondary to pathological myopia: 6month results. Br J Ophthalmol 2008; 92: 1035-1039.

22. Ikuno $Y$, Sayanagi K, Soga K, Sawa M, Tsujikawa M, Gomi $F$, et al. Intravitreal bevacizumab for choroidal neovascularization attributable to pathological myopia: oneyear results.Am J Ophthalmol 2009; 147: 94-100.

23. Chan WM, Lai TY, Liu DT, Lam DS. Intravitreal bevacizumab (Avastin) for myopic choroidal neovascularisation: 1year results of a prospective pilot study. Br J Ophthalmol 2009; 93: 150-154.

24. Gharbiya M, Allievi F, Mazzeo L, Gabrieli CB. Intravitreal bevacizumab treatment for choroidal neovascularization in pathologic myopia: 12-month results. Am J Ophthalmol 2009; 147: 84-89.

25. Ruiz-Moreno JM, Montero JA, Gomez-Ulla F, Ares S. Intravitreal bevacizumab to treat subfoveal choroidal neo- vascularisation in highly myopic eyes: 1-year outcome. $\mathrm{Br}$ J Ophthalmol 2009; 93: 448-451.

26. Silva RM, Ruiz-Moreno JM, Nascimento J, Carneiro A, Rosa P, Barbosaa A, et al. Short-term efficacy and safety of intravitreal ranibizumab for myopic choroidal neovascularization. Retina 2008; 28: 1117-1123.

27. Lai TY, Chan WM, Liu DT, Lam DS. Intravitreal ranibizumab for the primary treatment of choroidal neovascularization secondary to pathologic myopia. Retina 2009; 29: 750-756.

28. Konstantinidis L, Mantel I, Pournaras JA, Zografos L, Ambresin A. Intravitreal ranibizumab (Lucentis) for the treatment of myopic choroidal neovascularization. Graefes Arch Clin Exp Ophthalmol 2009; 247: 311-318.

29. Monés JM, Amselem L, Serrano A, Garcia M, Hijano M. Intravitreal ranibizumab for choroidal neovascularization secondary to pathologic myopia: 12-month results. Eye 2009; 23: 1275-1280.

30. Rosenfeld PJ. Intravitreal Avastin for choroidal neovascularisation in pathological myopia: the controversy continues. Br J Ophthalmol 2007; 91: 128-130.

31. Fung AE, Lalwani GA, Rosenfeld PJ, Dubovy SR, Michels $S$, Feuer WJ, et al. An optical coherence tomography-guided, variable dosing regimen with intravitreal ranibizumab (Lucentis) for neovascular age-related macular degeneration. Am J Ophthalmol 2007; 143: 566-583.

32. Regillo CD, Brown DM, Abraham P, Yue H, Ianchulev T, Schneider S, et al. Randomized, double-masked, shamcontrolled trial of ranibizumab for neovascular age-related macular degeneration: PIER Study year 1. Am J Ophthalmol 2008; 145: 239-248.

33. Spaide R. Ranibizumb according to need: a treatment for age-related macular degeneration. Am J Ophthalmol 2007; 143: 679-680. 